\title{
Fats and health: an evolving scenario
}


(C) Springer Healthcare - CEC Editore 2012

The multifaceted relationship between fat consumption and incidence of cardiovascular disease (namely, coronary events) has been fascinating researchers and lay people for decades. The prevailing current perception is that omega- 3 fatty acids are "good fats" whose consumption should be increased to optimise prevention. Conversely, other fats are "noxious" and should be reduced or eliminated from the diet: saturated fatty acids because they increase cholesterolaemia and, therefore, cardiovascular risk; omega- 6 fatty acids because they would biologically compete with omega-3, in this way reducing their healthful activities.

In truth, the picture is much more complex than this. Saturated fatty acids correlate with cardiovascular risk in ways that are less direct than was thought. As an example, dairy product consumption-despite their high levels of saturated fatsdoes not correlate (or is inversely correlated, hinting at protective activities) with cardiovascular risk. Omega- 6 fatty acids (typical of seed oils such as corn oil) are said to be consumed in excess in modern diets. However, based on the scant available information, levels of omega- 6 consumption are much lower than originally hypothesised. As an example, omega- 6 consumption in Italy represents only $4 \%$ of total calories, and is therefore below the lower limit of the range (5-10\%) set by international guidelines.

In this issue of Nutrafoods, Dariush Mozaffarian, from the prestigious Harvard School of Public Health and one of the world authorities in this field (he participates in large observational studies, such as the "Nurses" and the "Health Professionals"), clarifies the issue of whether omega- 6 fatty acids promote or actually reduce cardiovascular risk.

This issue extends beyond the scientific realm: information on the health role(s) of fats influences the formulation of health claims and labels, according to Regulation (EC) 1924/2006. Following up on this, this issue of Nutrafoods also features an article by Paola Testori Coggi, director general of DG SANCO, European Commission, who summarises the talk she gave at an international meeting on this topic, organised in Milan by the Nutrition Foundation of Italy (see page 75 for a brief meeting report).

In summary, the field of fats and health is rapidly developing, and food and nutraceutical companies should closely monitor it in the near future. 